\title{
Size dependent carrier thermal escape and transfer in bimodally distributed self assembled InAs/GaAs quantum dots
}

\author{
G. Muñoz-Matutano, ${ }^{1, a)}$ I. Suárez, ${ }^{2}$ J. Canet-Ferrer, ${ }^{1}$ B. Alén, ${ }^{2}$ D. Rivas, ${ }^{1}$ L. Seravalli, ${ }^{3}$ \\ G. Trevisi, ${ }^{3}$ P. Frigeri, ${ }^{3}$ and J. Martínez-Pastor ${ }^{1}$ \\ ${ }^{1}$ ICMUV, Instituto de Ciencia de Materiales, Universidad de Valencia, P.O. Box 2085, 46071 Valencia, Spain \\ ${ }^{2}$ IMM, Instituto de Microelectrónica de Madrid (CNM, CSIC), Isaac Newton 8, 28760 Tres Cantos, \\ Madrid, Spain \\ ${ }^{3}$ Istituto dei Materiali per IElettronica e il Magnetismo (CNR), Parco delle Scienze 37/a, I-43100 Parma, Italy
}

(Received 21 February 2012; accepted 15 May 2012; published online 25 June 2012)

\begin{abstract}
We have investigated the temperature dependent recombination dynamics in two bimodally distributed InAs self assembled quantum dots samples. A rate equations model has been implemented to investigate the thermally activated carrier escape mechanism which changes from exciton-like to uncorrelated electron and hole pairs as the quantum dot size varies. For the smaller dots, we find a hot exciton thermal escape process. We evaluated the thermal transfer process between quantum dots by the quantum dot density and carrier escape properties of both samples. (C) 2012 American Institute of Physics. [http://dx.doi.org/10.1063/1.4729315]
\end{abstract}

\section{INTRODUCTION}

Semiconductor quantum dots (QDs) represent a vast field of research for both fundamental physics and technology development. ${ }^{1,2}$ Yet, to use them as a real alternative to current technologies, they have to be fully functional at room temperature.

The main consequences of a temperature raise on the QD photoluminescence (PL) are the redshift of the semiconductor band gap, the broadening of the homogeneous linewidth, and the quenching of the PL intensity by thermal promotion of carriers to high energy levels. The redshift of energy transitions might be considered an advantage if we want to tune the emission of InAs QDs grown on GaAs to more interesting spectral windows $(1.3-1.5 \mu \mathrm{m})$. Homogeneous linewidth broadening is the direct consequence of the loss of coherence or dephasing. Although there are some fundamental QD properties that are less influenced by exciton-phonon interaction, like the QD hole spin coherence, a temperature raise will be detrimental in most coherent applications. ${ }^{3}$ Among all, thermally activated carrier escape is the most important problem for QD operation at high temperatures. A variety of carrier redistribution effects caused by temperature has been investigated in QD ensembles. ${ }^{4-15}$ It is commonly accepted that the sigmoidal evolution of the peak energy and full width at half maximum of the PL bands is due to carrier promotion from small QDs to larger ones. ${ }^{6}$ Therefore, quantum dot size distributions, carrier capture, relaxation, and re-trapping among QDs of different sizes had to be considered to model correctly the QD recombination dynamics. $^{2,6}$ These models reveal new effects like the competition between band narrowing by thermal escape processes and band broadening due to exciton-phonon interactions, ${ }^{7,16}$ or the role of the wetting layer (WL) continuous states as a mediator for carrier diffusion. $4,6,8,9,11,17-20$

\footnotetext{
${ }^{\text {a) }}$ Author to whom correspondence should be addressed. Electronic mail: guillermo.munoz@uv.es.
}

The thermally activated escape mechanism initiates the temperature dynamics and therefore has deserved a lot of attention in the past. It has been investigated attending to the available final states, i.e., QD excited states, ${ }^{15,21}$ wetting layer, 4,6,7,13,20,22 GaAs barrier, ${ }^{8,9,23}$ and impurity/defect levels. ${ }^{23-26}$ Thermal escape can be also investigated attending to the nature of the particles being promoted to a higher energy state. ${ }^{14}$ Depending on the model, the correlated (excitonic escape), $6,8,10,12,23$ the uncorrelated electron-hole pair (ambipolar escape), 4,9,14 or just one of the carriers (unipolar escape $)^{15}$ can be considered. Whether one or the other is appropriate in a particular sample depends on the barrier height and therefore might vary for QDs of given composition but different size. ${ }^{13}$

The QD areal density must be also considered as it might be comparable to the density of traps and defects competing for the same carriers. A superlinear evolution of the integrated intensity at high power excitation has been proposed as a sign of independent electron and hole capture and escape. ${ }^{8,9}$ However, the same effect was explained considering the saturation of temperature activated trap states in the barrier. ${ }^{10}$ Lobo et al. ${ }^{7}$ found that the efficiency on carrier transfer is limited by the rate of carrier transfer on the WL only for low density samples, and normal Arrhenius dependence has been found for high density samples. A similar result by Zhou et al. ${ }^{19}$ concluded that the WL might act as a quenching (transfer) channel for low (high) density samples while Torchynska ${ }^{23}$ has reported that the effective thermal activation energy might depend on the QD density.

Low density samples are also interesting because enable the study of single QDs and reveal new aspects of the carrier capture and escape mechanisms. ${ }^{8}$ Single QD emission spectra are characterized by the coexistence of exciton complexes of different charge state and particle number. ${ }^{27}$ They correspond to dynamical configurations, which cannot be explained with conventional rate equations for the ensemble averaged level occupations. ${ }^{28}$ The stochastic capture/escape processes can be better described using a random population model. Using such 
model, we have recently analyzed the excitation power dependence for a single QD finding that a small number of uncorrelated captures (electron alone, for instance) have a large impact in the micro-PL $\left(\mu\right.$-PL) spectra. ${ }^{29}$

In the following, we study the recombination dynamics in two InAs/GaAs QD samples, both containing a bimodal distribution of QDs: small quantum dots (SQD) and large quantum dots (LQD). Both samples have similar low QD densities but were grown with different design and growth parameters, in order to influence the QD size distribution. We present ensemble-PL and $\mu$-PL spectra recorded as a function of temperature as well as energy dependent time resolved PL (TRPL) transients. The data are analyzed using a rate equations model, which takes into account the size distribution and the thermal transfer within the SQD ensemble. As the QD size shrinks, we observe that the carrier escape mechanism changes from uncorrelated to excitonic and also find marked differences for the different WL morphologies.

\section{SAMPLES AND EXPERIMENTAL SET-UP}

MBE-grown structures consist of (i) $100 \mathrm{~nm} \mathrm{GaAs}$ buffer grown at $600{ }^{\circ} \mathrm{C}$, (ii) InAs QDs deposited by MBE at $0.01 \mathrm{ML} / \mathrm{s}$ at $\mathrm{Tg}$ growth temperatures, and (iii) a $20 \mathrm{~nm}$-thick GaAs cap layer grown by atomic layer MBE at $360^{\circ} \mathrm{C} .{ }^{30}$ In particular, sample II has been grown with $T_{g}=530^{\circ} \mathrm{C}$ and 2.0 ML resulting in a QD density of $25 \mathrm{QDs} / \mu \mathrm{m}^{2}$, while for the sample I the temperature was increased to $535^{\circ} \mathrm{C}$ and 2.50 ML to allow for a reduction of the QD density to 16.5 QDs $/ \mu \mathrm{m}^{2}$ and a change in QD size distribution (see Figs. $1(\mathrm{~b})$ and $1(\mathrm{c})$ ). It should be stressed that the coverage values indicate the amount of In supplied during growth and, due to the substantial In desorption occurring at such high temperatures, may not represent the effective deposited material. Further details about the growth procedure and in-depth discussion of the effect of different growth parameters on QD properties are available in Ref. 31. AFM characterization a)

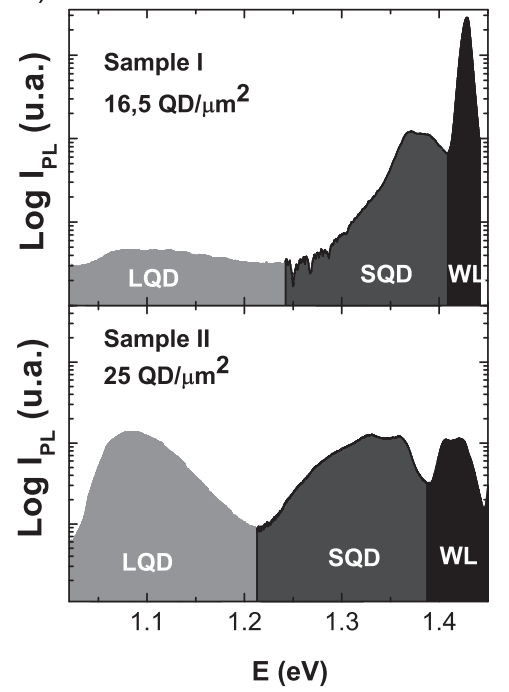

b)

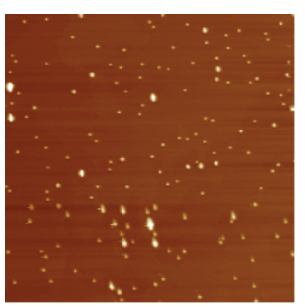

,

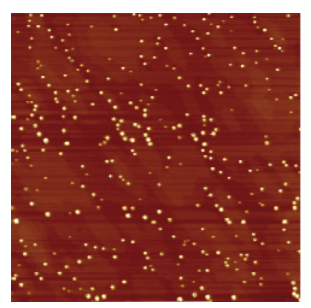

FIG. 1. (a) Top (lower) panel: Ensemble-PL from sample I (II) covered with three grey regions for the LQD, SQD, and WL optical emission. (b) $3 \times 3 \mu \mathrm{m}$ AFM picture from sample I. (c) $3 \times 3 \mu \mathrm{m}$ AFM picture from sample II. was performed on uncapped samples grown under similar conditions of samples studied by PL.

Conventional PL spectra as shown in Fig. 1 have been collected using a temperature variable He closed-cycle cryogenerator. A double monochromator selects the wavelength which we direct to a Si CCD or avalanche photodiode for CW or time resolved acquisition, respectively. The time resolution including the tunable $76 \mathrm{MHz}$ Ti:Saph pulsed laser is $\sim 400 \mathrm{ps}$ and has been deconvoluted to extract the decay lifetimes. The excitation laser wavelength was $790 \mathrm{~nm}(1.57 \mathrm{eV})$ and the excitation power density was $7 \mathrm{nw} / \mu \mathrm{m}^{2}$. The sample was also held in an immersion He cryostat to record $\mu$-PL spectra as a function of temperature using a fiber based microscope arrangement with single mode (multimode) excitation (collection) spot. With this configuration, we collect the emission from immediately captured excitons as well as from those diffusing through the WL before radiative recombination.

\section{EXPERIMENTAL RESULTS}

In this section, we present our results from temperature dependent ensemble-PL and TRPL and $\mu$-PL, for both samples I and II.

In Figure 1, we show the ensemble-PL spectra for both samples (a) and their corresponding AFM images (b, c). In both cases, the emission band is divided into three energy ranges: low and high energy QD emission bands associated with a bimodal size distribution and the highest energy emission band related to the WL. The low (high) energy emission bands correspond to the exciton recombination at LQD (SQD) families defined above. SQD emission from sample I is narrower and distributed close to the WL peak. The WL emission from sample I dominates over the QD emission and exhibits a single Gaussian shape (peaked at $\sim 1.428 \mathrm{eV}$ ). Meanwhile, in sample II, the WL emission intensity is weaker than the QD luminescence and exhibits a double peak structure (peaks at $\sim 1.406$ and $\sim 1.418 \mathrm{eV}$ ). This suggests that in sample I the WL has a larger two-dimensional (2D) character, while in sample II the WL has small islands with an inhomogeneous distribution on the WL thickness. ${ }^{15}$ Such WL morphological differences have been previously associated with different growth conditions as the continuous or interrupted growth protocols ${ }^{32}$ and with the sample misorientation. ${ }^{33,34}$ In our case, this effect may be related to the difference in temperature and coverage between the two samples. AFM characterization suggests that such WL differences between the samples may indeed be present (Figs. 1(b) and 1(c)).

Figures 2 and 3 show the temperature evolution of ensemble-PL for the SQD band (Figs. 2(a) and 2(b)) and for LQD band in both samples (Figs. 3(a) and 3(b)). The PL intensity quenching for the high energy SQD starts at low temperatures $(10-20 \mathrm{~K}$ and $30-40 \mathrm{~K}$ for samples I and II, respectively), while for LQD bands the PL intensity quenching starts at higher temperatures $(70-80 \mathrm{~K}$ and $120-140 \mathrm{~K}$ for samples I and II, respectively), as expected for a thermally activated process. ${ }^{4-6}$ SQD and LQD bands in sample I also exhibit an increase of the integrated intensity with temperature as it is shown on the Arrhenius plots at Figures 3(c). 

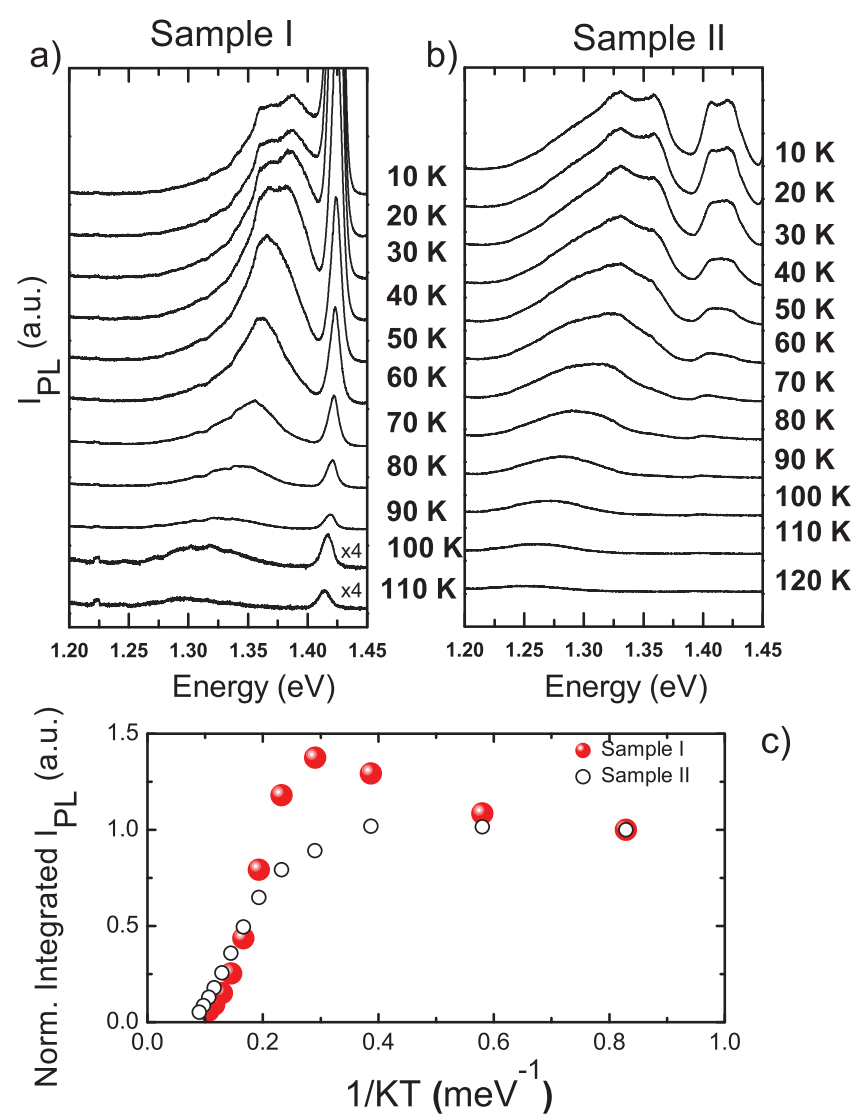

c)
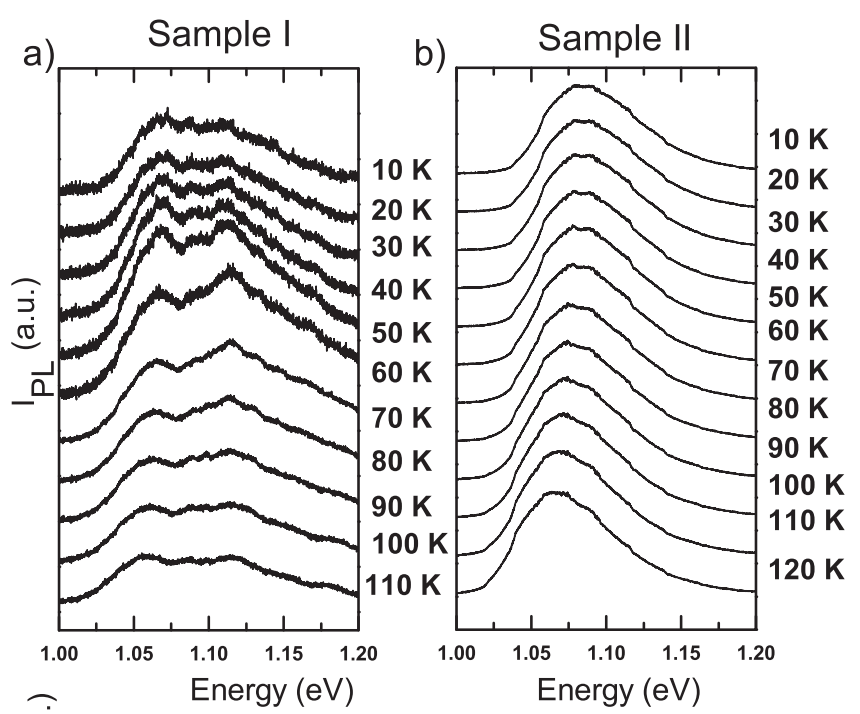

ક๋

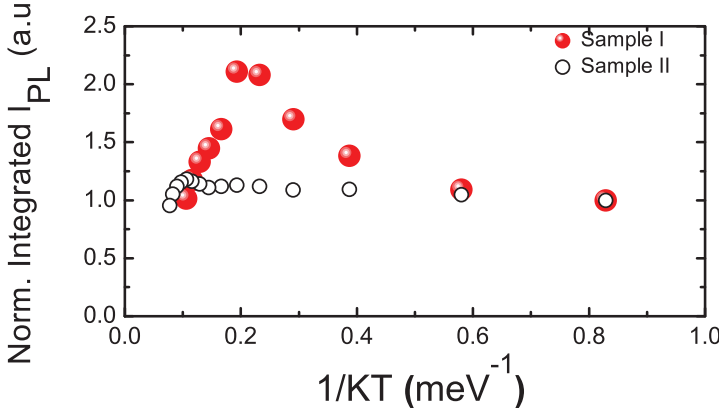

c)
FIG. 2. Ensemble-PL temperature dependence from SQD bands from samples I (a) and II (b). (c) Arrhenius plot for the integrated intensity of the entire SQD band, normalized to the $10 \mathrm{~K}$ initial integrated intensity. It was used $7 \times 10^{-3} \mu \mathrm{W} / \mu \mathrm{m}^{2}$ as $790 \mathrm{~nm}$ excitation source.

This PL-temperature trend is an accepted signature of a carrier thermal transfer. ${ }^{7,13,22,35-38}$

However, there are some particularities which must be taken into account. The integrated intensity from sample I SQD band increases at intermediate temperatures. This effect must be fulfilled by an extra exciton injection channel. It could be assumed some QD to QD transfer mechanism, which is justified by the ensemble-PL linewidth temperature evolution $^{6}$ (Fig. 2(a)). Nevertheless, the extra exciton injection in the SQD sample I band comes from WL carrier diffusion at intermediates temperatures $(\sim 40 \mathrm{~K})$. Therefore, as temperature rises, carriers at WL suffer an activated carrier channel loss. Sample II SQD band temperature evolution has a different trend, as it is shown in Figure 2(c). The results did not show an extra excitonic injection in the Arrhenious plot, i.e., it is not present any significant increasing of the integrated intensity. As a result, sample II SQD band evolves following conventional thermal escape and transfer through WL states with WL diffusion process activated even at $10 \mathrm{~K}$. This will be discussed in more detail in Sec. IV.

Contour plots at Figures 4(a) and 4(b) show the $\mu$-PL temperature evolution from samples I and II. Here, we can compare the $\mu$-PL thermal evolution between two samples characterized by SQD bands whose energy distance to the $\mathrm{WL}$ is appreciably different. The regions where single SQD is strongly affected by the thermal escape are indicated with two white straight lines on both contour plots (Figs. 4(a) and 4(b)).
FIG. 3. Ensemble-PL temperature dependence from LQD bands from samples I (a) and II (b). (c) Arrhenius plot for the integrated intensity of the entire LQD band, normalized to the $10 \mathrm{~K}$ initial integrated intensity. It was used $7 \times 10^{-3} \mu \mathrm{W} / \mu \mathrm{m}^{2}$ as $790 \mathrm{~nm}$ excitation source.

The temperature evolution of the $\mu$-PL in four single QDs $\left(\mathrm{A}^{\prime}, \mathrm{B}^{\prime}, \mathrm{C}^{\prime}\right.$, and $\mathrm{D}^{\prime}$ for sample I and $\mathrm{A}, \mathrm{B}, \mathrm{C}$, and $\mathrm{D}$ for sample II) is marked in Figures 4(a) and 4(b), whereas the evolution of their intensity is represented by Arrhenius plots in Figures 4(c)-4(f). We observe $\mu$-PL quenching above $\sim 30 \mathrm{~K}$ for the SQD band in sample I (Fig. 4(d)) and above $\sim 60 \mathrm{~K}$ in sample II (Fig. 4(f)). The integrated intensity with temperature below $60 \mathrm{~K}$ increases in both samples, but it occurs at higher temperatures in sample II and has a stronger effect in sample I. This is in good correspondence with the larger mean SQD-WL energy difference on sample II and the observed carrier thermal injection described in the above ensemble-PL temperature analysis.

Finally, we labeled $S_{e}-S_{h}$ and $P_{e}-P_{h}$ in both Figures 4(a) and 4(b) as the fundamental and first excited optical transitions from LQD bands. Both samples show thermal transfer to the first LQD excited states ( $\mathrm{B}^{\prime}$ and $\mathrm{B}$ peaks at Figures 4(a) and 4(b), and their Arrhenious plots at Figures 4(c) and 4(e)). The effect has been shown previously in ensemble-PL studies ${ }^{19}$ and could be used as a thermal excitation to generate molecular coupling between $P_{e}-P_{h}$ LQD and resonant $S_{e}-S_{h}$ SQD levels in a similar procedure than in the exciton to exciton thermal coupling ${ }^{48}$ and transfer. ${ }^{49}$ More details concerning excited state assignation and evaluation could be found in Ref. 27. In summary, by this micro-PL study we demonstrate the presence of thermal carrier promotion to SQD and LQD bands in both samples. 


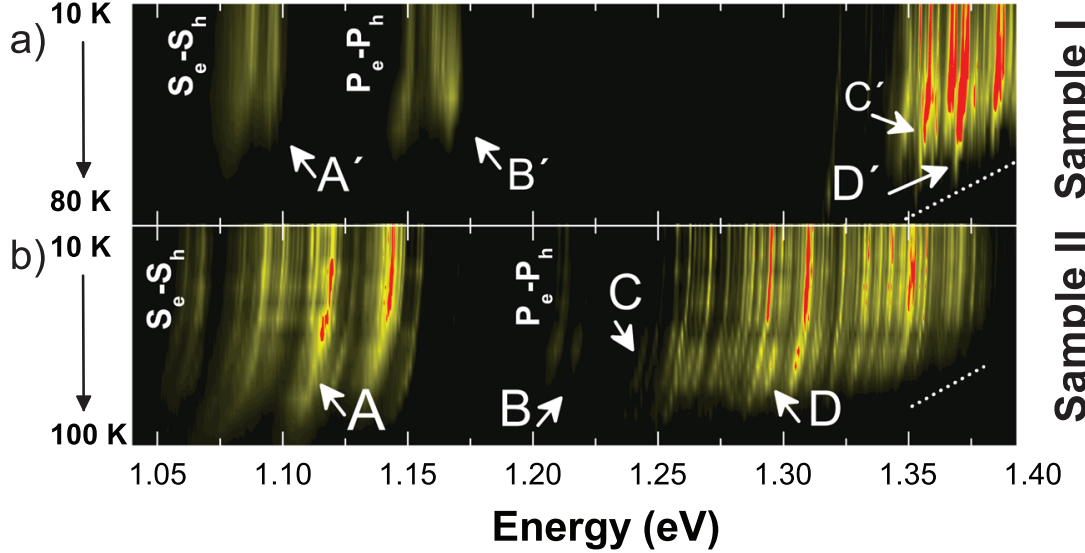

LQD SQD
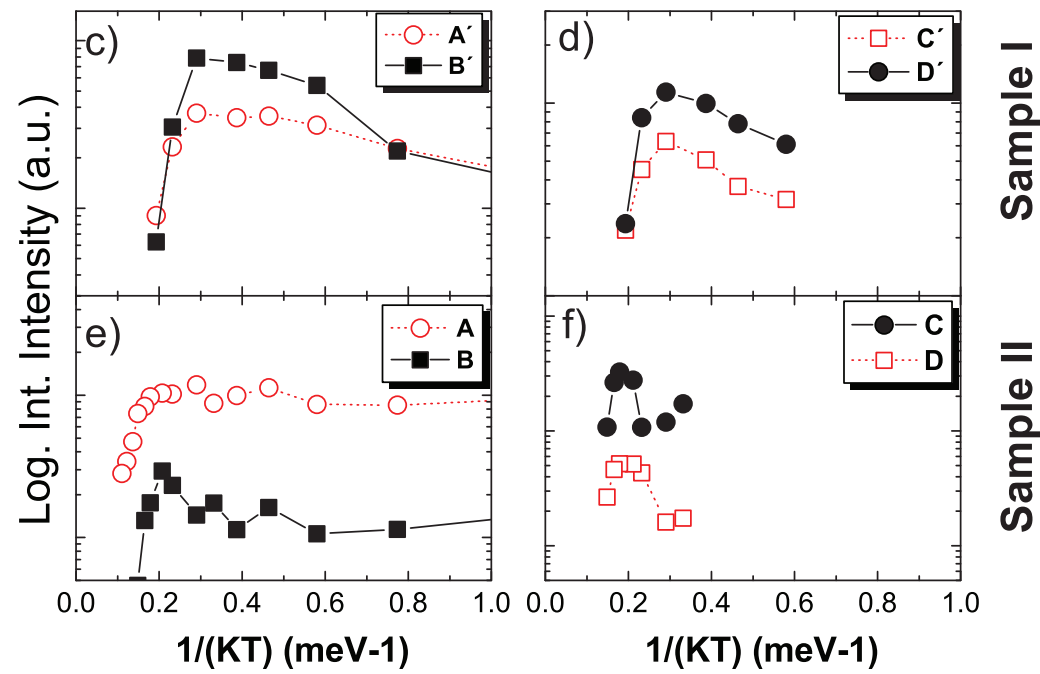

FIG. 4. Contour plots: $\mu$-PL vs temperature for samples I (a) and II (b). Arrhenius plots for the QDs labeled in (a) from the LQD distribution in samples I (c) and II (e). Arrhenius plots for the QD labeled in (b) from the SQD distribution in samples I (d) and II (f).
Further insight can be obtained from the temperature dependence of the PL decay time within the SQD distribution on both samples. PL transients are reproduced by single monoexponential decays. The extracted time constants are summarized in the upper panels in Figures 5(a) and 5 (b), as a function of the emission energy and temperature. In both samples, above a certain temperature the decay time falls to a sub-nanosecond scale due to the activation of non-radiative channels. ${ }^{4,13,37,39-41}$ The observed temperatures at which PL decay times begin to decrease in Figure 5 are in good correspondence with the QD Arrhenius plots. As observed for the integrated intensity, the PL decay time increases in the low temperature range. Both effects have been reported in the literature ${ }^{4,13,39,40,42-44}$ and might be attributed here to thermal transfer of carriers ${ }^{4,39,42}$ among nearby QDs.

\section{THERMAL CARRIER ESCAPE AND TRANSFER}

In this section, we analyze both thermal carrier escape and transfer on the basis of the experimental results just described. We have adapted a rate equation model to our system, based on the previous description from Yang et al., ${ }^{4}$ to study the QD size dependence on the carrier thermal escape. Yang et al. ${ }^{4}$ proposed the integration of the escape rate on the whole QD energy band. Using similar definitions and approaches, we propose to divide the entire ensemble into a number of single QD energies (sizes) using a finite set of delta like QD density of states. We obtain an energy dependent estimation of the carrier escape process, and, therefore, an evaluation of the correlated/uncorrelated escape nature as function on the QD size. This model has a principal shortcoming: We lost most of the inhomogeneous QD information. Therefore, in this study we neglect the ensemble-PL lineshape temperature evolution.

\section{A. Thermal escape}

We have restricted this study to the SQD family, as these QDs have smaller size and thus thermal escape represents the main carrier loss mechanism in the low-medium temperature range $(10-100 \mathrm{~K})$. The model is valid within the excitonic approach at low excitation density. We assume that the QD population is well described by excitons. ${ }^{29}$

Figure 6 shows the carrier dynamics scheme proposed in this work. The whole feeding process is given by the $\mathrm{G}$ parameter, representing the direct excitonic pumping to the WL. Excitons at the WL can recombine with a rate $q_{r}$, thermally promoted to the GaAs barrier or captured within the QD ensemble. At high enough temperatures, the excitons at the $\mathrm{i}-\mathrm{QD}$ of the distribution can promote to the WL with a rate $e_{i}$. Under thermal equilibrium conditions, energy independent capture rate (C) and i-QD escape rate $\left(e_{i}\right)$ are related to each other through 
a)

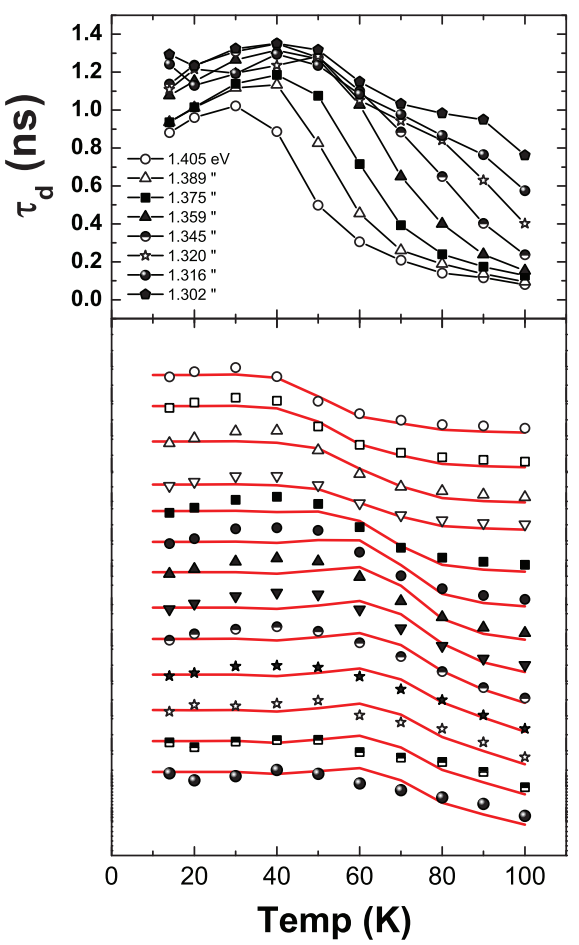

$\frac{e_{i}}{C} \propto \exp \left(-\frac{\nu_{i} \Delta E_{Q D-i}}{k T}\right)$

where $\Delta E_{Q D-i}=E_{W L}-E_{Q D-i}$ is the energy difference between the WL and the i-QD excitonic ground state, $k$ is the Boltzmann constant, and $T$ is the lattice temperature. The parameter $\nu_{i}$ represents the ratio between the actual activation energy $\left(E_{i}^{a}\right)$ and $\Delta E_{Q D-i}\left(\nu_{i}=\frac{E_{i}^{a}}{\Delta E_{Q D-i}}\right)$. This parameter has been used to classify the carrier escape nature. ${ }^{14}$ The value $\nu=1$ is associated with correlated (excitonic) escape, while $\nu<0.5$ and $\nu=0.5$ to unipolar and uncorrelated pair escape, respectively. In their model, Yang et al. ${ }^{4}$ obtain a single $\nu$ value, independent of the QD emission energy. An energy dependent $\nu$ value $\left(\nu_{i}=\nu_{i}\left(E_{i}\right)\right)$ leads to the following rate equations for WL population $(M(t))$ and i-QD populations $\left(N_{i}(t)\right)$ :

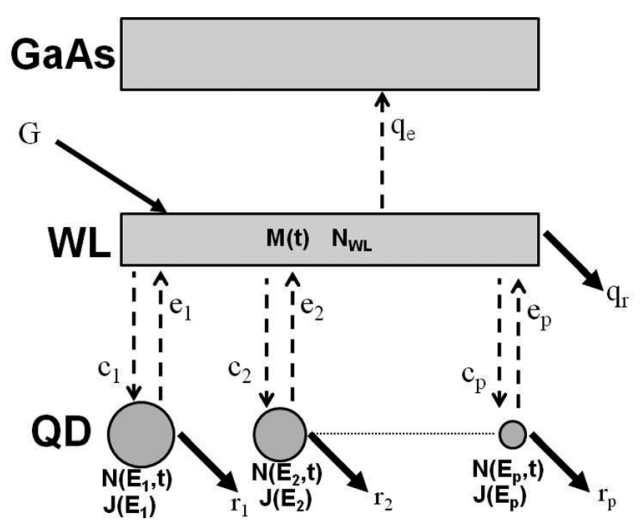

FIG. 6. Carrier dynamics scheme proposed for the InAs/GaAs QD system and corresponding for the rate equation model from Eq. (2).
FIG, 5. Upper panels: Single layer representation for decay time temperature dependence from samples I (a) and II (b). Lower panels: same decay time evolution represented on multilayer plot from samples I (a) and II (b). Red continuous lines correspond to the numerical fitting results from a balance equation model. where $q_{e}$ is the WL to GaAs escape rate, including its temperature dependence as $q_{e}=q_{e, 0} \exp \left(-\frac{\Delta E_{B}}{K T}\right)\left(\Delta E_{B}=E_{G a A s}\right.$ $-E_{W L}$ is the energy difference between GaAs and WL band edges and $q_{e, 0}$ is a constant). Subscripts $i$ and $p$ indicate the individual energy and the total number of energies considered in the problem, respectively. $r_{i}$ is the effective recombination rate from the $\mathrm{i}-\mathrm{QD}$. Each $c_{i}$ is calculated by the product $c_{i}=J_{i} * C$, where $J_{i}$ is the normalized density of states for the i-QD energy, obtained from the $10 \mathrm{~K}$ ensemblePL lineshape. ${ }^{6}$ The model reproduces quite well the experimental data from the TRPL by fitting the parameters $q_{e, 0}, C$, and $\nu_{i}$, as it is shown in Figure 5. The input parameters are the number of QD energies $(p)$, the SQD-QD areal density $\left(N_{S Q D}\right)$, WL exciton mass $\left(m_{W L}\right)$, GaAs-WL energy difference $\left(\Delta E_{B}\right)$, the WL effective recombination rate $\left(q_{r}\right)$, and the effective decay rate from all i-QDs $\left(r_{i}\right)$.

Table I summarizes all input values found or assumed for the parameters of the model in both samples. The areal density of SQD, $N_{S Q D}$, is estimated both from AFM images and from low power ensemble-PL integrated intensity analysis. The exciton effective mass at the WL, $m_{W L}$, is assumed to be $0.4 m_{0}{ }^{4}$ The WL transition energies are at 1.428 and $1.418 \mathrm{eV}$, giving $\Delta E_{B}$ takes values of 88 and $98 \mathrm{meV}$ in samples I and II, respectively. The WL emission in sample II is composed by two PL lines. This suggests that there could be an additional thermal escape rate and hence $q_{r}$ is modeled as $q_{r}=q_{r, 0}+q_{r, 1} * e^{\left(-\frac{\Delta E_{W L}}{K T}\right)}$. The first term is the experimental 
TABLE I. Parameters used to calculate PL decays.

\begin{tabular}{lcc}
\hline \hline Parameter & Sample I & Sample II \\
\hline $\mathrm{P}$ & 15 & 16 \\
$q_{r}$ & $q_{r, 0}^{E x p}(T)$ & $q_{r, 0}^{E x p}(T)+150 * e^{\frac{-15(\mathrm{meV})}{\mathrm{KT}}}$ \\
$\Delta E_{B}$ & $0.088 \mathrm{eV}$ & $0.109 \mathrm{eV}$ \\
$m_{W L}$ & $0.40 * m_{0}$ & $0.40 * m_{0}$ \\
$N_{S Q D}$ & $13 \times 10^{12} \mathrm{~m}^{-2}$ & $15 \times 10^{12} \mathrm{~m}^{-2}$ \\
$\mathrm{R}$ & 0.881 to $1.293 \mathrm{~ns}^{-1}$ & 1.292 to $1.290 \mathrm{~ns}^{-1}$ \\
$q_{e, 0}$ & $1 \times 10^{6} \mathrm{~ns}^{-1}$ & $1 \times 10^{6} \mathrm{~ns}^{-1}$ \\
$1 / C$ & $83 \mathrm{ps}$ & $83 \mathrm{ps}$ \\
$v_{i}=v_{i}\left(E_{S Q D}\right)$ & See. Fig. $8(\mathrm{a})$ & See. Fig. $8(\mathrm{a})$ \\
\hline \hline
\end{tabular}

WL recombination rate measured at $10 \mathrm{~K}$ and takes the value of $14.2 \mathrm{~ns}^{-1}$. The second term takes into account the thermal escape, being $q_{r, 1}$ a fitting parameter and $\Delta E_{W L}$ the observed energy difference between both WL PL lines. The QD effective recombination rate $\left(r_{i}\right)$ is composed by the sum of radiative and non-radiative terms. The QD radiative recombination rate is obtained from the inverse of the measured PL decay time at $10 \mathrm{~K}$. The activation energy of the thermal carrier capture into defect states has been set to $\sim 20 \mathrm{meV},{ }^{25,26}$ and hence $r_{o, j} * e^{\left(-\frac{20}{K T}\right)} \quad(\mathrm{j}=\mathrm{I}, \quad \mathrm{II})$, where $r_{0, j=I} \sim 15$ and $r_{0, j=I I} \sim 1$ are obtained for samples I and II. Such a high $\frac{r_{0, I}}{r_{0, I I}}$ ratio might be related to the different effective coverages of the two samples: The larger In coverage of sample I might induce ripening of QDs with associated nucleation of structural defects, as widely described in Ref. 45. $1 / C$ is equal to $83 \mathrm{ps}$ in both samples.

The main output parameter is the energy dependent $\nu_{i}$ values plotted in Figure 7(a). We observe how $\nu_{i}$ decreases with the QD size in both samples with almost the same trend, reflecting the carrier escape character. Correlated (or excitonic) escape $(\nu \sim 1)$ is found for SQD whose emission occurs between $E_{W L}-E_{Q D}=40-70 \mathrm{meV}$, as shown in Figure 7 (a). In sample II, $\nu$ goes down to 0.8 as the energy difference increases. These observations are in good correspondence with the results from Schulz et al., ${ }^{13}$ where in bimodally distributed samples they found excitonic escape in the high energy PL band and uncorrelated pair escape in the low energy PL band.

We also find that $2>\nu>1$ for the highest QD emission energies in both samples. These $\nu$ values cannot be explained by direct thermal escape to upper levels $\left(W L_{H H}(\nu=1)\right.$, $W L_{L H}(\nu \sim 3)$, GaAs $\left.(\nu \sim 4)\right)$. In this range, the activation energy $\nu_{i} \times\left(E_{W L}-E_{Q D_{i}}\right)$ remains constant and equal to $\sim 36 \mathrm{meV}$ (GaAs LO phonon energy). Within the current model, this means that thermal activation for the smallest QDs occurs through absorption of a single LO phonon and resonant injection of a hot exciton $(K \neq 0)$ into the WL continuous state. Assuming a parabolic dispersion for the WL with $m=0.4 m_{0}, K$ varies between 0.4 and $0.11 \mathrm{~nm}^{-1}$ for $\Delta E_{Q D-i}$ between 22 and $35 \mathrm{meV}$. These values of $K$ are of the same order than the inverse of the exciton localization length, $1 / L_{X} \sim 0.2 \mathrm{~nm}^{-1}$, with $L_{X} \sim 5 \mathrm{~nm}$ typical for small QDs. ${ }^{46}$

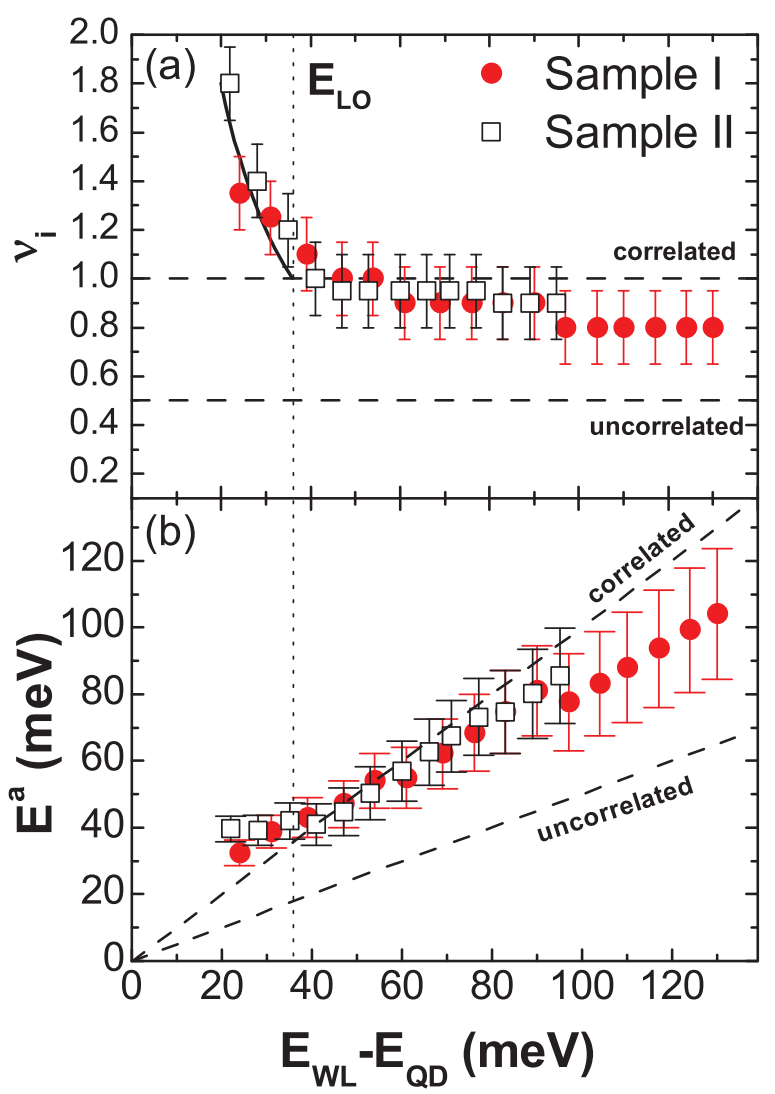

FIG. 7. (a) Energy dependence for the $v_{i}$ output parameter. Black continuous line corresponds to $v_{i}=\hbar \omega_{L O} /\left(E_{W L}-E_{Q D}\right)$. (b) Energy dependence for the activation energy $\left(E^{a}\right)$ derived from the $v_{i}$ parameter. Doted lines correspond to the uncorrelated and correlated carrier escape trends.

\section{B. Thermal transfer}

In this section, we analyze the QD carrier thermal transfer through WL states in both samples.

To determine the in-plane motion of carriers in the WL and their trapping rate, areal density has to be compared with the diffusion length. In the literature, the problem has been divided into two extreme cases ${ }^{18,20}$ : high and low QD density samples. In the first case, carrier trapping from WL to QDs is limited by QD to QD distance $\left(d_{Q D-Q D}\right)$. For high QD density samples, carriers are trapped in the QDs before they can radiatively recombine in the WL, i.e., $d_{Q D-Q D}$ distance is smaller than the 2D WL effective diffusion length $\left(L_{D}\right)$. In these circumstances, QD emission is stronger than WL emission. ${ }^{20}$ This is the case of sample II, as it is shown in Figure 1.

In the second case, carrier diffusion within the WL is not limited by $d_{Q D-Q D}$ distances, as these distances are similar or larger than $L_{D}$. The WL optical emission is pronounced and dominates over the QD optical emission. ${ }^{20}$ This is the case of sample I, as it is shown in Figure 1. Following the model proposed by Ohmori et al., ${ }^{20}$ we estimate the $L_{D}$ value of sample I studying the temperature dependence of the relative integrated intensity between SQD and WL bands. This model is based on the proportionality between $L_{D}$ and the QD/WL emission intensity ratio $\left(\beta=\frac{I_{Q D}}{I_{W L}}\right)$. The $L_{D}$ values deduced from the $\beta$ coefficient at different temperatures in sample I are $\approx 90(230) \mathrm{nm}$ at 10 (40) K. These values are similar to those found in other studies on InAs QDs. ${ }^{18,20,47}$ 


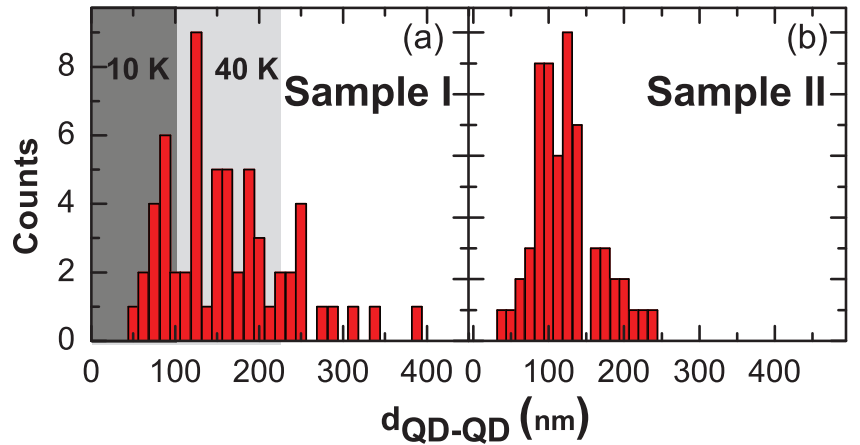

FIG. 8. Interdot distance $\left(d_{Q D-Q D}\right)$ statistics for samples I (a) and II (b) measured from the AFM images from Figure 1. The two grey shaded areas in (a) indicate sample I $L_{D}$ values at 10 and $40 \mathrm{~K}$.

Figure 8 shows a statistical analysis of $d_{Q D-Q D}$ from AFM images of both samples in Figure 1. The most probable interdot distance $d_{Q D-Q D}$ is $\sim 125$ and $\sim 110 \mathrm{~nm}$ in samples I and II, respectively. We have added two gray shaded areas in Figure 8 (a) to indicate the $d_{Q D-Q D}$ distances covered by $L_{D}$ at 10 and $40 \mathrm{~K}$, as obtained from the approach described above for sample I. The carrier diffusion in the WL at $10 \mathrm{~K}$ is able to connect a small fraction of neighbour QDs $\left(L_{D}^{10 \mathrm{~K}}=90 \mathrm{~nm}<d_{Q D-Q D}^{\text {mean }}=125 \mathrm{~nm}\right)$. However, the diffusion at $40 \mathrm{~K}$ would reach most of the $d_{Q D-Q D}$ distances $\left(L_{D}^{40 \mathrm{~K}}=230 \mathrm{~nm}>d_{Q D-Q D}^{\text {mean }}=125 \mathrm{~nm}\right)$. It could be expected that thermal carrier transfer between QDs should be more efficient in sample II, as its $d_{Q D-Q D}$ distribution is centered at a lower value. However, the smaller energy difference between the SQD and WL states in sample I allows that a large number of thermally activated carriers can spatially diffuse in the WL and be transferred to other QDs at relatively low temperatures $(\sim 40 \mathrm{~K})$. For this reason, a more efficient thermal transfer is expected in sample I at low temperatures, which is in concordance with our ensemble and $\mu$-PL results from Sec. III.

\section{CONCLUSION}

In summary, we have measured temperature dependence of the ensemble-PL, $\mu$-PL, and TRPL in two bimodal distributed InAs/GaAs self assembled QDs samples. The observed increase of the integrated intensity and the decay times for both samples are tentatively attributed to thermal carrier transfer through the WL state. We have derived a rate equation approach to study the thermal escape through the analysis of the energy dependent $\nu$ parameter. Our data show excitonic escape for the smaller dots emitting close to the WL energy. This correlated escape character is relaxed as the QD size becomes larger. Finally, we have shown how WL carrier diffusion length vs inter quantum dot distances plays a relevant role in the QD thermal carrier transfer process present in these samples.

\section{ACKNOWLEDGMENTS}

We gratefully acknowledge the financial support of the Generalitat Valenciana, Comunidad Autnoma de Madrid and the Spanish Ministry Projects Nos. PROMETEO/2009/074,
S2009ESP-1503 and TEC-2008-06756-C03-03, TEC201129120-C05-04/01. One of the authors D. Rivas thanks the Ministry of Science for his FPI fellowship. The AFM characterization has been carried out at CIM, University of Parma, Italy.

${ }^{1}$ D. Bimberg, Semiconductor Nanostructures (Springer Verlag, Berlin, 2008).

${ }^{2}$ Z. M. Wang, Self Assembled Quantum Dots (Springer Science + Business Media LLC, 2008).

${ }^{3}$ B. Gerardot, D. Brunner, P. Dalgarno, P. Öhberg, S. Seidl, M. Kroner, K. Karrai, N. Stoltz, P. Petroff, and R. Warburton, Nature (London) 451, 441 (2008).

${ }^{4}$ W. Yang, R. Lowe-Webb, H. Lee, and P. Sercel, Phys. Rev. B 56, 13314 (1997).

${ }^{5}$ R. Heitz, I. Mukhametzhanov, A. Madhukar, A. Hoffmann, and D. Bimberg, J. Electron. Mater. 28, 520 (1999).

${ }^{6}$ S. Sanguinetti, M. Henini, M. G. Alessi, M. Capizzi, P. Frigeri, and S. Franchi, Phys. Rev. B 60, 8276 (1999).

${ }^{7}$ C. Lobo, R. Leon, S. Marcinkevicius, W. Yang, P. Sercel, X. Liao, J. Zou, and D. Cockayne, Phys. Rev. B 60, 16647 (1999).

${ }^{8}$ E. L. Ru, J. Fack, and R. Murray, Phys. Rev. B 67, 245318 (2003).

${ }^{9}$ P. Dawson, O. Rubel, S. Baranovskii, K. Pierz, P. Thomas, and E. Göbel, Phys. Rev. B 72, 235301 (2005).

${ }^{10}$ S. Sanguinetti, D. Colombo, M. Guzzi, E. Grilli, M. Gurioli, L. Seravalli, P. Frigeri, and S. Franchi, Phys. Rev. B 74, 205302 (2006).

${ }^{11}$ Y. Mazur, B. Liang, Z. Wang, D. Guzun, G. Salamo, Z. Zhuchenko, and G. Tarasov, Appl. Phys. Lett. 89, 151914 (2006).

${ }^{12}$ T. Torchynska, J. C. Espinosa, L. Borkovska, S. Ostapenko, M. Dybiec, O. Polupan, N. Korsunska, A. Stinz, P. Eliseev, and K. Malloy, J. Appl. Phys. 101, 024323 (2007).

${ }^{13}$ W. Schulz, R. Robach, M. Reischle, G. Beirne, M. Bommer, M. Jetter, and P. Michler, Phys. Rev. B 79, 035329 (2009).

${ }^{14}$ G. Gélinas, A. Lanacer, R. Leonelli, R. Masut, and P. Poole, Phys. Rev. B 81, 235426 (2010).

${ }^{15}$ A. Lévesque, P. Desjardins, R. Leonelli, and R. Masut, Phys. Rev. B 83, 235304 (2011).

${ }^{16}$ M. Smirnov, V. Talalaev, B. Novikov, S. Sarangov, N. Zakharov, P. Werner, U. Gösele, J. Tomm, and G. Cirlin, Phys. Status Solidi B 247, 347 (2010).

${ }^{17}$ L. Brusaferri, S. Sanguinetti, E. Grilli, M. Guzzi, A. Bignazzi, F. Bogani, L. Carraresi, M. Colocci, A. Bosacchi, P. Frigeri et al., Appl. Phys. Lett. 69, 3354 (1996).

${ }^{18}$ S. Hinooda, S. Loualiche, B. L. N. Bertu, M. Paillard, X. Marie, and T. Amand, Appl. Phys. Lett. 78, 3052 (2001).

${ }^{19}$ X. Zhou, Y. Chen, J. Liu, G. Zhou, X. Ye, B. Xu, and Z. Wang, J. Phys. D: Appl. Phys. 43, 295401 (2010).

${ }^{20}$ M. Ohmori, P. Vitushinskiy, and H. Sakaki, Appl. Phys. Lett. 98, 133109 (2011).

${ }^{21}$ X. Zhou, Y. Chen, X. Ye, B. Xu, and Z. Wang, J. Appl. Phys. 109, 113540 (2011).

${ }^{22}$ Y. Zhang, C. Huang, F. Liu, B. Xu, J. Wu, Y. Chen, D. Ding, W. Jiang, X. Ye, and Z. Wang, J. Appl. Phys. 90, 1973 (2001).

${ }^{23}$ T. Torchynska, J. Appl. Phys. 104, 074315 (2008).

${ }^{24}$ G. Saint-Girons and I. Sagnes, J. Appl. Phys. 91, 10115 (2002).

${ }^{25}$ L. Seravalli, M. Minelli, P. Frigeri, S. Franchi, G. Guizzetti, M. Patrini, T. Ciabattoni, and M. Geddo, J. Appl. Phys. 101, 024313 (2007).

${ }^{26}$ L. Seravalli, G. Trevisi, P. Frigeri, S. Franchi, M. Geddo, and G. Guizzetti, Nanotechnology 20, 275703 (2009).

${ }^{27}$ G. Muñoz-Matutano, B. Alén, J. Martínez-Pastor, L. Seravalli, P. Frigeri, and S. Franchi, Nanotechnology 19, 145711 (2008).

${ }^{28}$ M. Grundmann, R. Heitz, D. Bimberg, J. Sandmann, and J. Feldmann, Phys. Status Solidi B 203, 121 (1997).

${ }^{29}$ J. Gomis-Brescó, G. Muñoz-Matutano, B. Alén, J. Martínez-Pastor, L. Seravalli, P. Frigeri, G. Trevisi, and S. Franch, New J. Phys. 13, 023022 (2011).

${ }^{30}$ F. Briones, L. Gonzalez, and A. Ruiz, Appl. Phys. A 49, 729 (1989).

${ }^{31}$ G. Trevisi, L. Seravalli, P. Frigeri, and S. Franchi, Nanotechnology 20, 415607 (2009).

${ }^{32}$ A. Balzarotti, Nanotechnology 19, 505701 (2008).

${ }^{33}$ V. Evtikhiev, V. Tokranov, A. Kryganovskii, A. Boiko, R. Suris, and A. Titkov, J. Cryst. Growth 201, 1154 (1999).

${ }^{34}$ M. Xu, Y. Temko, T. Suzuki, and K. Jacobi, Surf. Sci. 580, 30 (2005).

${ }^{35}$ H. Htoon, H. Yu, D. Kulik, J. Keto, O. Baklenov, A. Holmes, B. Streetman, and C. Shih, Phys. Rev. B 60, 11026 (1999). 
${ }^{36}$ Y. Mazur, X. Wang, Z. Wang, G. Salamo, M. Xiao, and H. Kissel, Appl. Phys. Lett. 81, 2469 (2002).

${ }^{37}$ W. Ouerghui, J. Martínez-Pastor, J. Gómis, A. Melliti, M. Maaref, D. Granados, and J. García, Eur. Phys. J.: Appl. Phys. 35, 159 (2006).

${ }^{38}$ X. Lu and J. Vaillancourt, Semicond. Sci. Technol. 26, 045013 (2011).

${ }^{39}$ A. Melliti, M. Maaref, F. Hassen, M.Hjiri, H. Maaref, J. Tignon, and B. Sermage, Solid State Commun. 128, 213 (2003).

${ }^{40}$ J. Gomis, J. Martínez-Pastor, B. Alén, D. Granados, J. García, and P. Roussignol, Eur. Phys. J. B 54, 471 (2006).

${ }^{41}$ M. Gurioli, A. Vinattieri, M. Zamfirescu, M. Colocci, S. Sanguinetti, and R. Nötzel, Phys. Rev. B 73, 085302 (2006).

${ }^{42}$ H.-S. Kwack, Y.-H. Cho, J.-D. Song, W.-J. Choi, and J.-I. Lee, J. Appl. Phys. 106, 123524 (2009)
${ }^{43}$ L. Kong, Z. Feng, Z. Wu, and W. Lu, J. Appl. Phys. 106, 013512 (2009).

${ }^{44}$ H. Lee, M.-Y. Ryu, and J. Kim, J. Appl. Phys. 108, 093521 (2010).

${ }^{45}$ P. Frigeri, L. Nasi, M. Prezioso, L. Seravalli, G. Trevisi, E. Gombia, R. Mosca, F. Germini, C. Bocchi, and S. Franchi, J. Appl. Phys. 102, 083506 (2007).

${ }^{46}$ B. Alén, J. Martínez-Pastor, D. Granados, and J. M. García, Phys. Rev. B 72, 155331 (2005)

${ }^{47}$ E. Dupuy, D. Morris, N. Pauc, V. Aimez, M. Gendry, and D. Drouin, Appl. Phys. Lett. 94, 022113 (2009).

${ }^{48}$ B. Gerardot, S. Strauf, M. de Dood, A. Bychkov, A. Badolato, K. Hennessy, E. Hu, D. Bouwmeester, and P. Petroff, Phys. Rev. Lett. 95, 137403 (2005).

${ }^{49}$ B.-L. Liang, Z.-M. Wang, X.-Y. Wang, J.-H. Lee, Y. Mazur, C.-K. Shih, and G. Salamo, ACS Nano 2, 2219 (2008). 